\title{
The order-chaos-order sequence in the spring pendulum
}

\author{
J.P. van der Weele*, E. de Kleine \\ Centre for Theoretical Physics, University of Twente, PO Box 217, 7500 AE Enschede, The Netherlands
}

\begin{abstract}
We study the motions of a spring pendulum as a function of its two control parameters (the ratio of the spring and pendulum frequencies, and the energy). It is shown that in the limits for very small and very large parameter values the dynamics of the spring pendulum is predominantly regular, while at intermediate parameter values the majority of initial conditions lead to chaotic trajectories. Thus, upon varying the parameters from small to large values one typically witncsses a transition from order to chaos and back to order again. Similar order-chaos-order sequences are observed in many other dynamical systems, and the spring pendulum is a representative example.

In this context, we also discuss the phenomenon for which the spring pendulum is famous, namely the to-and-fro transfer between spring-and pendulum-like behaviour when the spring frequency is (approximately) twice the pendulum frequency. This turns out to play an important role in the order-chaos-order sequence.
\end{abstract}

\section{Introduction}

In physical systems depending on one or more variable parameters it is often observed that the dynamical behaviour is extremely complicated at intermediate values of the parameters, but relatively simple if the parameters are either very small or very large. For example, a weather-vane flies in a complicated way at average wind speeds, but it simply hangs down when it is calm, and it is stretched horizontally in a uniform gale. Another example is provided by two nonlinearly coupled Toda oscillators [1]. As a function of the total energy and the coupling parameter, the motion of the oscillators may change from regular to chaotic and back to regular again. The same kind of behaviour is found in the generalized Hénon-Heiles problem [2] and in the spring pendulum [3-5]. In each of these examples one witnesses, upon varying the control parameters (wind force, coupling strength, etc.) a so-called

\footnotetext{
* Corresponding author. 
order-chaos-order sequence. That is, the system goes from a situation in which most of the phase space is occupied by regular orbits to a situation in which most of the phase space is filled with chaos, and then back to a regular situation again. It should be emphasized from the start that this transition does not concern one specific orbit. but rather the net effect of all the orbits taken together.

To most people the first part of the sequence (order to chaos) seems quite natural, but the second part (the reversal to order) often comes as a surprise. It is the purpose of the present paper to show that it is nevertheless perfectly logical. To this end we take a closer look at the example of the spring pendulum, depicted in Fig. 1. which (by its elegant simplicity) is well suited to demonstrate some general aspects of the order chaos order sequence. It may be pointed out that in some systems a similar sequence is observed, but with order in the middle and chaos at both ends. i.e. a chaos-order-chaos sequence. An example of this is found in the coupled oscillators of Ref. [6]. This may seem totally different at first, but in fact it is merely a variation upon the sane theme, and the results of the spring pendulum are, with necessary changes made, also applicable to those systems.

The spring pendulum has two degrees of freedom (the spring direction $r$, and the pendulum direction $\varphi$ ) but only one constant of the motion, namely, the total energy. This means that the system is non-integrable and is liable to exhibit chaotic behaviour. Indeed it does so when the control parameters of the system, to be introduced in the next section, have intermediate values. However, if the parameter values are either very small or very large the chaos disappears.

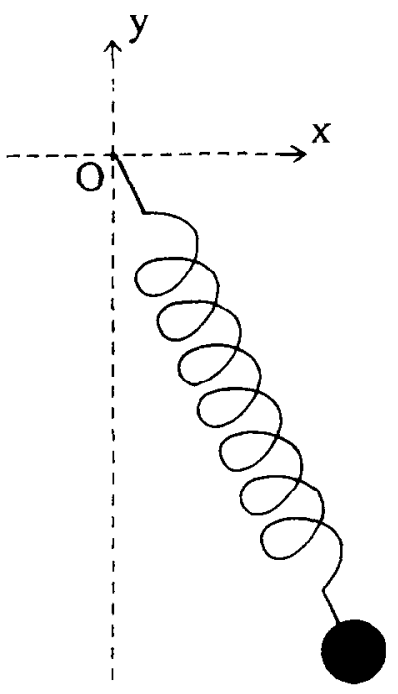

Fig. 1. The spring pendulum. 
The paper is organized as follows. In Section 2, we define the system, write down its equations of motion and specify the two control parameters $\mu$ and $R$. In Section 3, we study how the dynamical behaviour of the spring pendulum depends on these parameters, making a survey of the $(\mu, R)$ plane and identifying the main types of motion, which include oscillations as well as rotations. In Section 4, the cases for very small and very large parameter values are treated, and we explain the absence of chaos in these limits. In Section 5 we turn to the region of intermediate parameter values and explain the abundance of chaos. Finally, in Section 6, we discuss our results.

\section{The equations of motion}

The spring pendulum, depicted in Fig. 1, consists of a massless spring of rest length $\ell_{0}$ and stiffness $k$, with a mass $m$ attached to it. In its equilibrium position the spring will be stretched, due to the weight $m g$, to a length

$$
\ell_{\mathrm{c}}=\ell_{0}+\frac{m g}{k}
$$

The assumption that the spring has no mass, which is meant to keep our model as simple as possible, is quite an idealization and one might worry if it is not an oversimplification. For one thing, a spring mass significantly diminishes the frequency of the (dominant) spring vibration. For another thing, a massive spring introduces an infinite amount of extra degrees of freedom into the problem, and one actually has to deal with a whole spectrum of vibrations within the spring. We will come back to this in Section 6.

Our (idealized) spring pendulum has two degrees of freedom. It combines the behaviour of a spring, with angular frequency

$$
\omega_{\mathrm{s}}=\sqrt{\frac{k}{m}}
$$

with that of a pendulum, with (for small amplitudes)

$$
\omega_{\mathrm{p}}=\sqrt{\frac{g}{\ell_{\mathrm{c}}}}=\sqrt{\frac{g}{\ell_{0}+m g / k}} .
$$

One can describe the system either in Carlesian or in polar coordinates. We will use both, just as the occasion demands, since some features are best described in terms of $x$ and $y$ and others in $r$ and $\varphi$. In Cartesian coordinates, and in the absence of dissipation, the Lagrangian reads:

$$
L-\frac{1}{2} m\left(\dot{x}^{2}+\dot{y}^{2}\right)-m g y-\frac{1}{2} k\left(\sqrt{x^{2}+y^{2}}-f_{u}\right)^{2},
$$


and from this we readily obtain the equations of motion:

$$
\ddot{x}=-\frac{k}{m} x\left(1-\frac{t_{0}}{\sqrt{x^{2}+y^{2}}}\right), \quad \ddot{y}=-g-\frac{k}{m} y\left(1-\frac{t_{0}}{\sqrt{x^{2}+y^{2}}}\right) .
$$

The equivalent equations in polar coordinates are given in the Appendix. It should be noted that we have just now made another substantial idealization: we ignore dissipation. This is (again) to keep the model as simple as possible. We shall come back to dissipation in Section 6.

The spring pendulum (without dissipation) has one constant of the motion, namely the total energy:

$$
E=\frac{1}{2} m\left(\dot{x}^{2}+\dot{y}^{2}\right)+m g y+\frac{1}{2} k\left(\sqrt{x^{2}+y^{2}}-t_{0}\right)^{2} .
$$

In Fig. 2 we have plotted the contour plot of the potential energy for typical values of $m, k$ and $\ell_{0}$ (while $y=9.81 \mathrm{~m} / \mathrm{s}^{2}$ ). From this plot or from common sense we see that the energy is minimal when the spring pendulum is hanging downward in its equilibrium position, with $x=0$ and $y=-\left(t_{0}+m g / k\right)$.

$$
E_{\mathrm{min}}=-m g\left(1_{0}+\frac{m g}{k}\right)+\frac{1}{2} k\left(\frac{m g}{k}\right)^{2}=-m g\left(t_{0}+\frac{1}{2} \frac{m g}{k}\right) .
$$

The energy cannot become smaller than this. On the other hand, there is no upper bound to the energy. One can vary value of $E$, from $E_{\mathrm{min}}$ to infinity, by taking different initial conditions. Instead of the energy itself one may also take the following

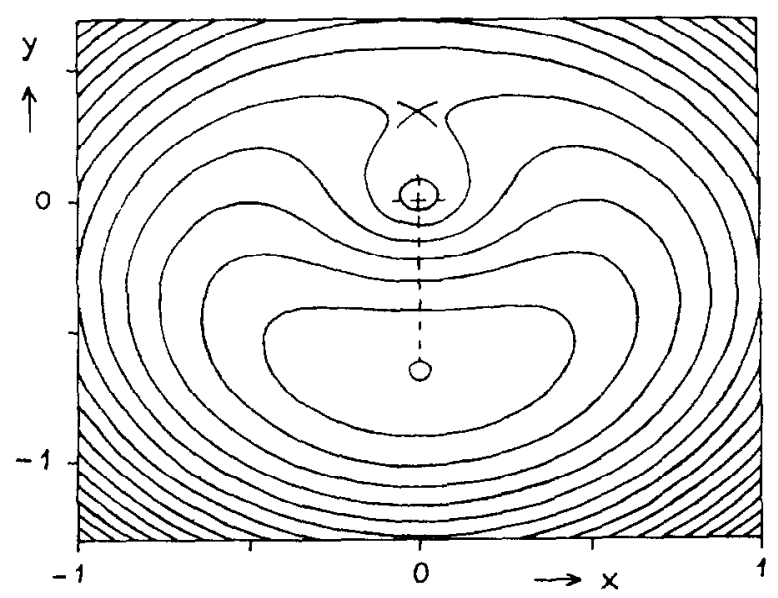

Fig. 2. Contour plot of the potential energy, with $m=0.25 \mathrm{~kg}, k=15 \mathrm{~N} / \mathrm{m}$ and $/ 0=0.5 \mathrm{~m}(\mathrm{i} . \mathrm{e} . ., \mu=4.06)$. The pendulum (dashed line) is depicted in its equilibrium position, representing the minimal energy $F_{\text {min }}$. The (unstable) upward equilibrium is denoted by a cross. 
dimensionless parameter:

$$
R \equiv-\frac{E}{E_{\min }}
$$

The range of this parameter, the so-called energy ratio, is -1 to infinity.

Apart from the energy (or energy ratio) the spring pendulum has a second important parameter, namely:

$$
\mu=1+\frac{k t_{0}}{m g}
$$

which, for small amplitudes of the spring pendulum, is equal to the squared ratio of the spring and pendulum frequencies: $\mu \equiv \omega_{\mathrm{s}}^{2} / \omega_{\mathrm{p}}^{2}$. Another useful relation is $\ell_{\mathrm{c}}=\mu f_{0} /(1-\mu)$, or $\mu=\left(1-\ell_{0} / \ell_{\mathrm{e}}\right)^{-1}$.

Just like $R$, the parameter $\mu$ is dimensionless. It can take on any value between 1 and infinity. A value of special interest is $\mu=4$, or $\omega_{\mathrm{s}}=2 \omega_{\mathrm{p}}$, in which case the spring and pendulum modes are in autoparametric resonance. This gives rise to a beautiful exchange of energy between the spring motion and the pendulum motion. Indeed, the behaviour of the spring pendulum at $\mu=4$ is so remarkable that almost all authors restrict themselves to this value [3-5, 7-13]. In doing so, however, one misses all the nice physics at other values of $\mu$.

The energy-ratio $R$ and the frequency-ratio $\mu$ are the two control parameters of the system. In the next section we examine how the dynamical behaviour depends on them.

\section{A survey of the parameter plane}

To begin with, in Fig. 3 we present the parameter plane, with $\mu$ along the horizontal axis and $R$ along the vertical axis. The shading in the central region of this plane indicates that chaos is abundant there, and the absence of shading in the outer region corresponds to an absence of chaos. If one takes for instance the points marked a to $f$, the phase space will be quite regular in $a, b, e$ and $f$ but contain a lot of chaos in $c$ and $d$. In Figs 4(a)-4(f) we illustrate this with a series of Poincaré sections at these six points. The sections ${ }^{1}$ show the $(y, \dot{y})$ plane at the moments when the spring pendulum passes through the line $x=0$ with positive horizontal velocity $(\dot{x} \geqslant 0)$. The abundance of chaos in Figs. 4(c) and 4(d) is evident, and so is the regularity of the other four Poincaré sections.

\footnotetext{
${ }^{1}$ It should be noted that in the numerical computations $\omega_{\mathrm{s}}^{2}$ is invariably taken to be $100 \mathrm{~s}{ }^{2}$, which implies that $\mu$ is varied solely by means of the rest length $\ell_{0}$ (in practice one would rather vary the stiffness $k$ or the mass $m$, see Section 4) and that $\omega_{\mathrm{p}}^{2}=g / t_{\mathrm{e}}=100 / \mu$, or $t_{\mathrm{e}}=0.0981 \mu$. That is why the center of the section, which lies in the neighbourhood of $(y, y)=\left(-\ell_{e}, 0\right)$, shifts to the left for growing $\mu$.
} 
Thus, if we travel through the shaded region, from small parameter values to large ones (or vice versa), we typically witness an order chaos-order sequence. As stated already in the introduction, the order chaos-order sequence is the net effect of a thousand minor changes and bifurcations at the level of the individual orbits. But it may be added that some orbits are more important than others. The main orbits can be recognized in the Poincare sections as conspicuous center points, denoted by the letters A, B, etc.. which persist over a large range of parameter values and form the

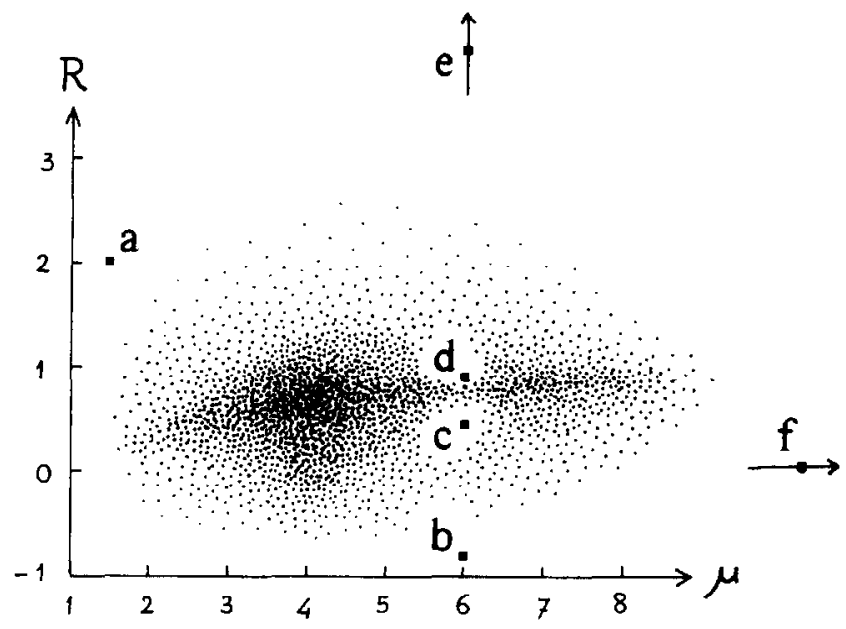

Fig. 3. The $(\mu, R)$ plane. The points a f correspond to ligs $4(a)-(f)$, and the shading is a measure of the abundance of chasos.

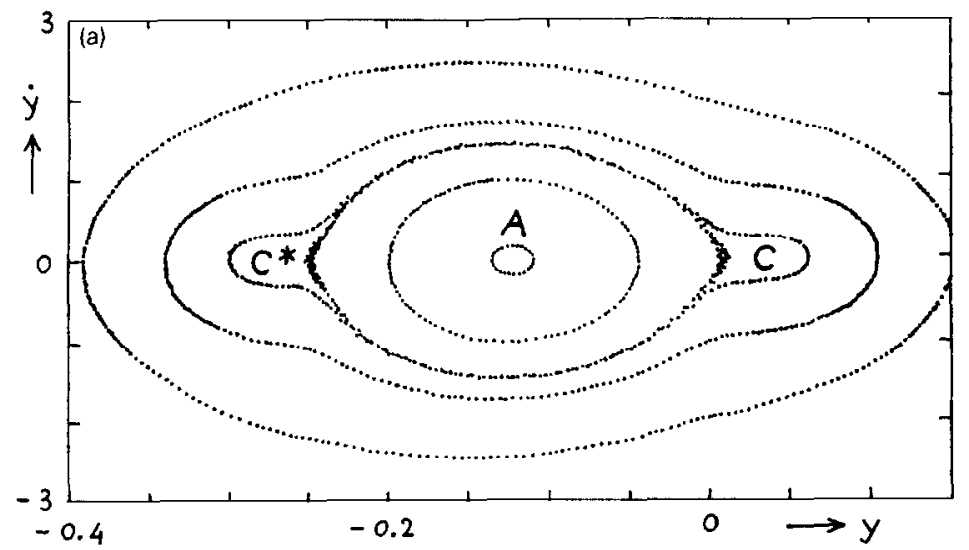

Fig. 4. Poincare sections through the phase space of the spring pendulum, showing ( $y$, $y)$ at the moments when $x=0$ and $\dot{x} \geqslant 0$. The values of the control parameters $(\mu, R)$ are as follows: (a) $(1.5,2.08)$, (b) $(6,-0.95)$. (c) $(6,0.38)$, (d) $(6,0.95)$, (c) $(6,189)$ and (f) $(100,0.021)$. Note the difference in scalc between the various plots. 

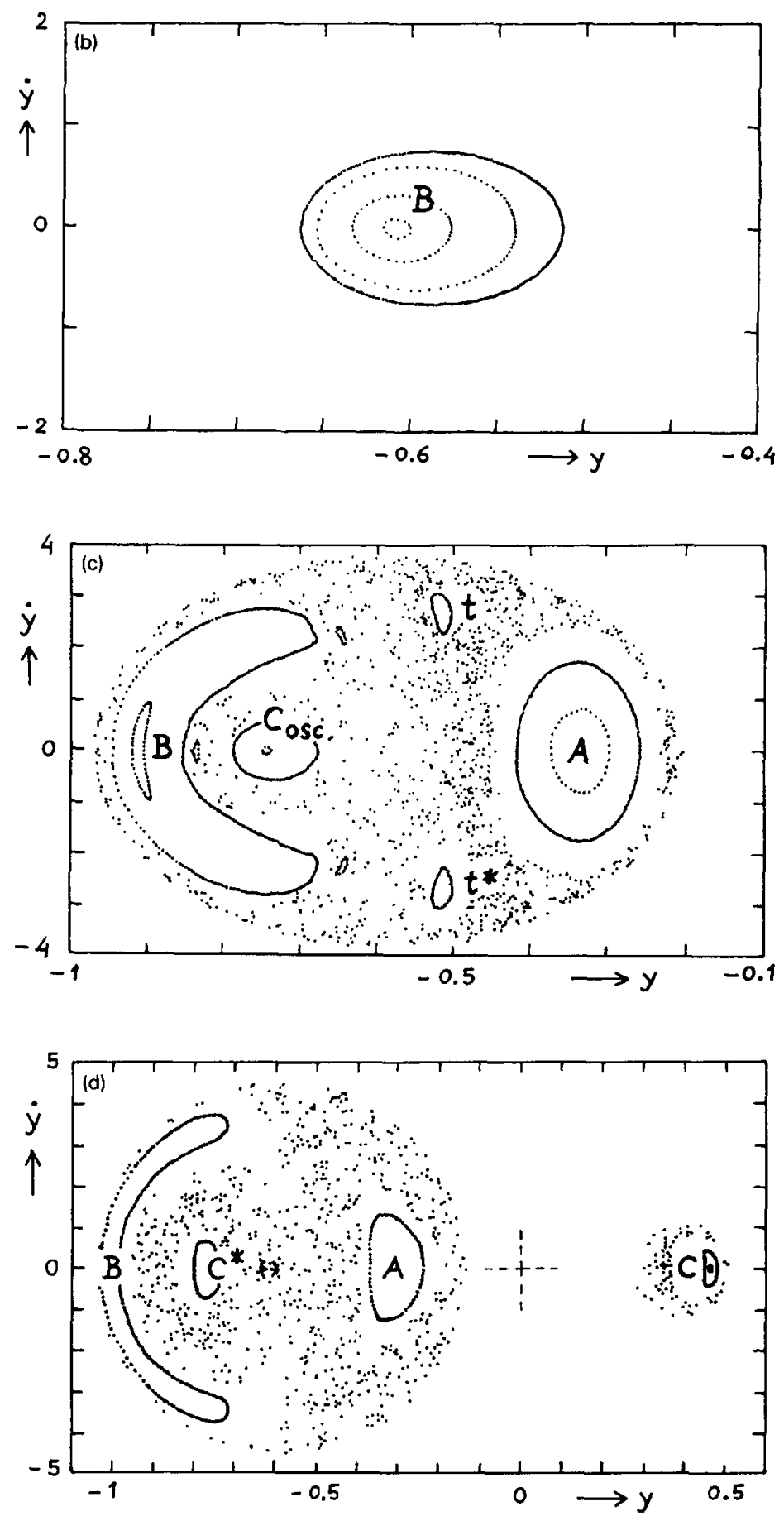

Fig. 4. Continued. 

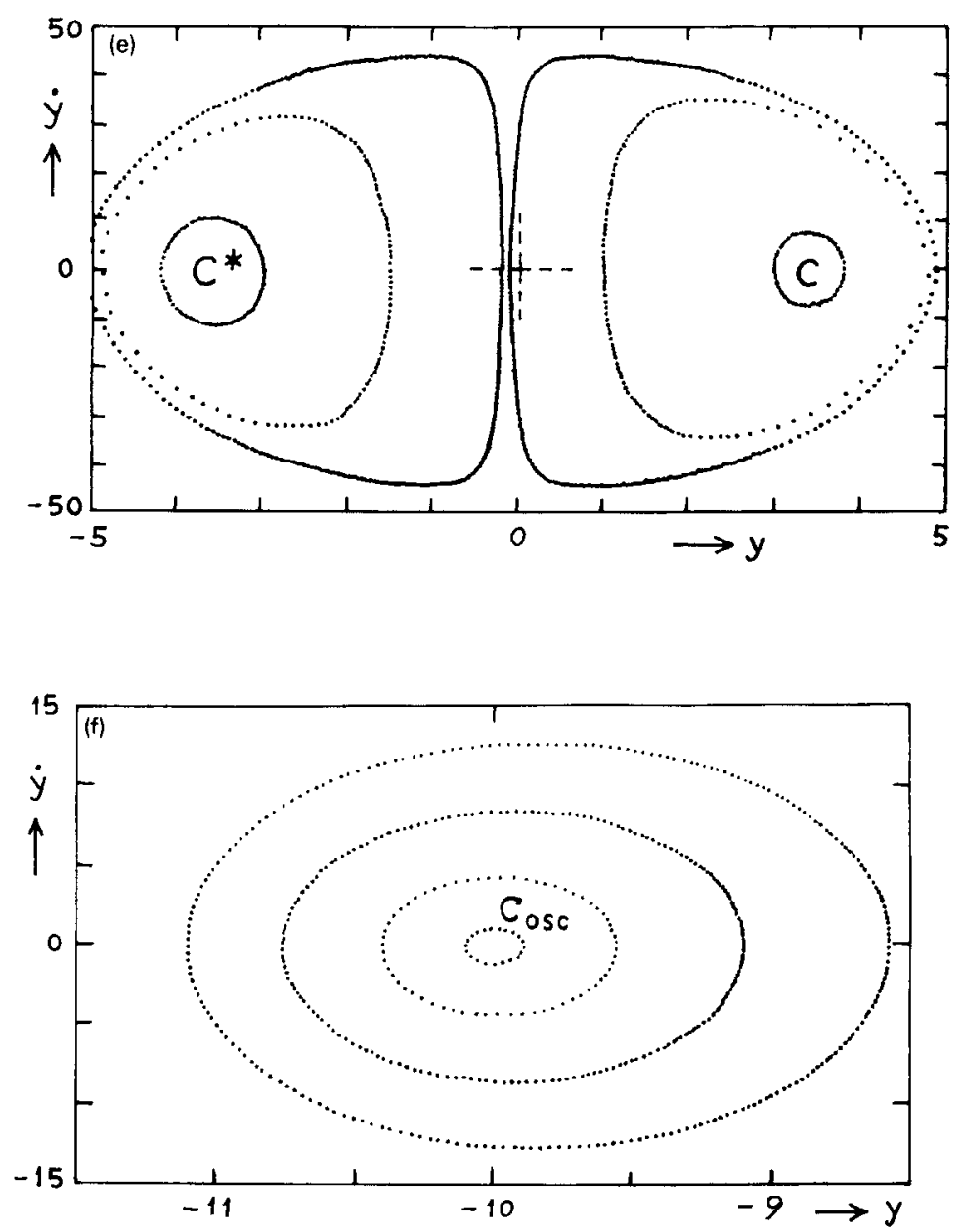

Fig. 4. Continued.

skclcton of the dynamics, in the sense that the other orbits are organized around them. In Fig. 5 we have sketched the form of the main motions in the ordinary $(x, y)$ space. We see that they include both oscillations and rotations, as was to be expected for a pendulum system; oscillations are the dominant (in fact the only) type of motion for small values of $R$ and rotations are the dominant type for large $R$. The A oscillation has the shape of a hill, and its counterpart is the valley-shaped $B$ oscillation. Closcly related to $B$ is the $C_{o s e}$ oscillation; indeed, in the Poincare sections $C_{o s e}$ is seen to be born (via a saddle-center bifurcation) within the embrace of $B$. In its turn, $C_{\text {osc }}$ paves the way for the rotations $C$ and $C^{*}$. In fact, as soon as $C_{o s e}$ has enough energy to make a closed loop it simply becomes $\mathrm{C}$ or $\mathrm{C}^{*}$. We will frequently come across these main motions in the following pages. 

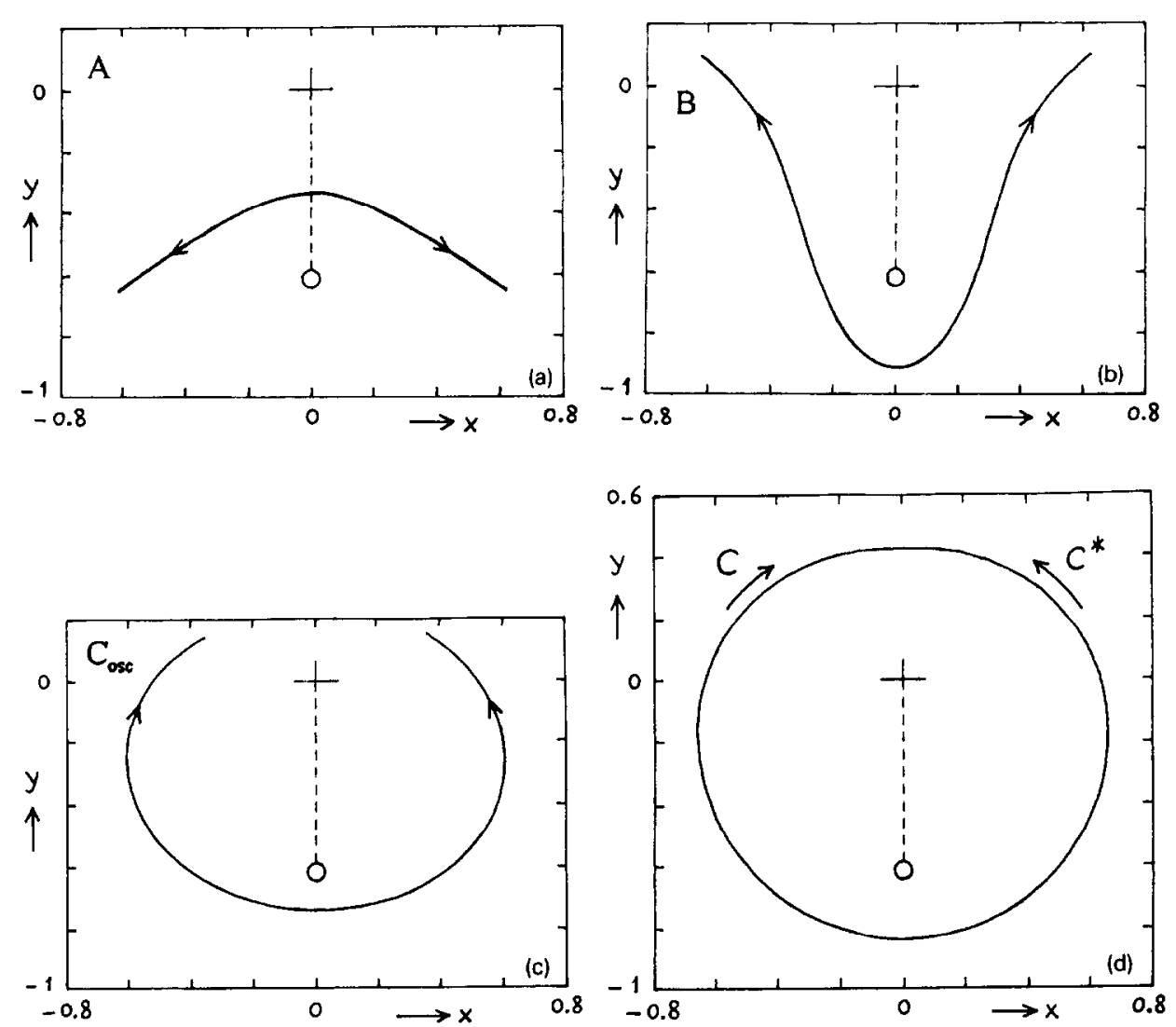

Fig. 5. A gallery of the main types of motion of the spring pendulum with (a) a hill oscillation $\mathrm{A}$, (b) a valley oscillation $\mathrm{B},(\mathrm{c})$ a horseshoe oscillation $\mathrm{C}_{\mathrm{osc}}$, and (d) a clockwise/counter-clockwise rotation $\left(\mathrm{C} / \mathrm{C}^{*}\right)$. The point of support and the downward equilibrium position have been included to guide the eye.

Around the main motions the spring pendulum can perform myriads of satellite motions. If the main motions are the skeleton of the dynamics, these satellite motions are the flesh on the bones. They can be periodic, quasi-periodic or chaotic, and it is here that the true richness of the dynamics comes out. It is impossible to represent this in a few pictures but for a simple illustration, in Fig. 6 we present two satellite motions (one periodic and one chaotic).

In order to see some examples of the "thousand minor changes and bifurcations", which together are responsible for the order-chaos-order sequence, we now focus on two orbits. In Fig. 7 we take a certain non-symmetrical oscillation $t$, a satellite of $\mathrm{B}$, which disintegrates via period doubling bifurcations upon entering the shaded region of the $(\mu, R)$ parameter plane. The orbit is shown, together with 

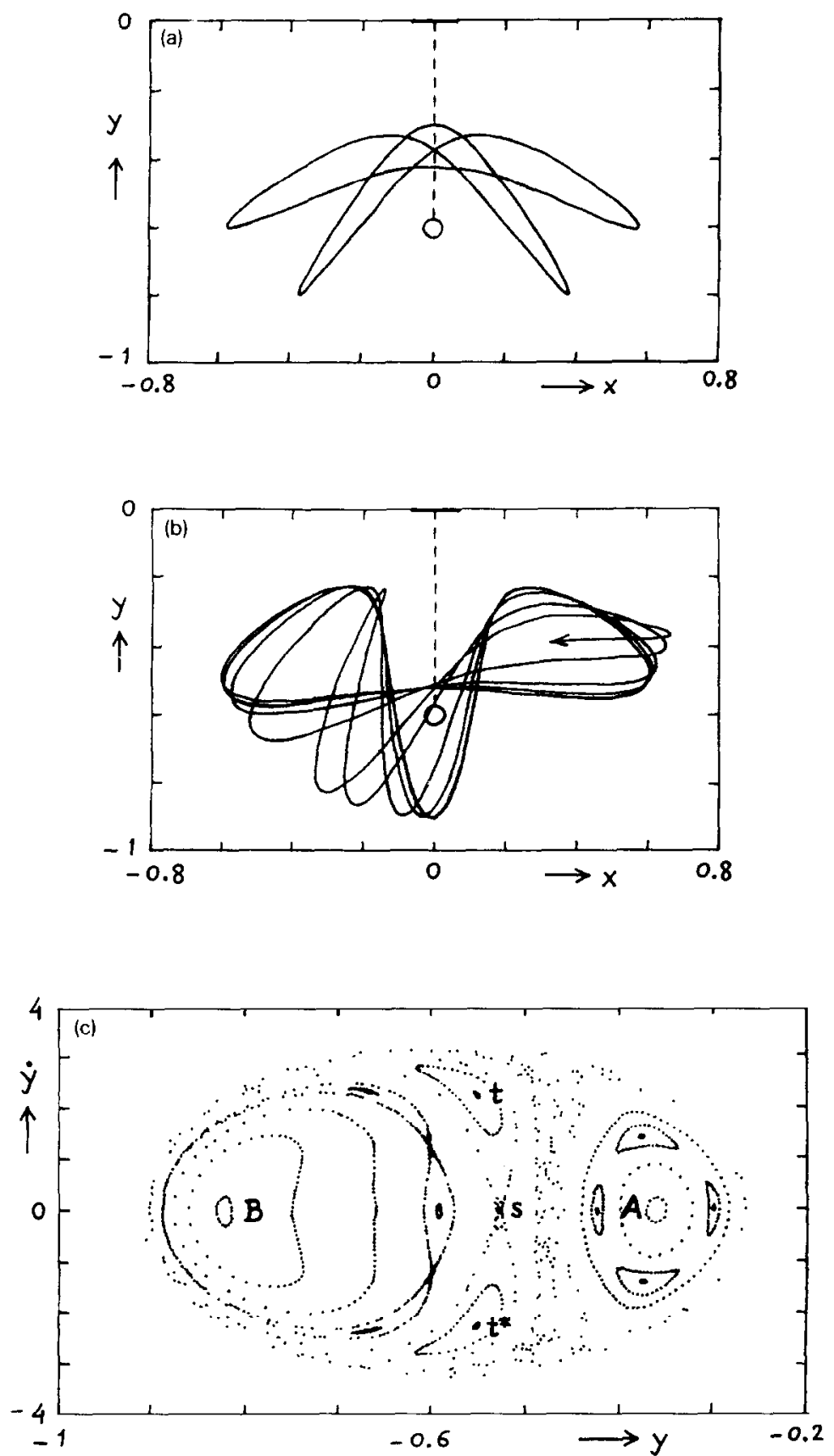

Fig. 6. (a) A regular satellite of the A motion. It corresponds to the four small islands around $A$ in the Poincaré section of (c). (b) A chaotic satellite of the B motion. The initial conditions were chosen close to point $\mathrm{s}$ in (c), which corresponds to an unstable, symmetric oscillation. (c) Poincaré section at $\mu=6, R=0$. The letters $t$ and $t^{*}$ denote the two non-symmetrical oscillations which wo follow in Fig. 7. 

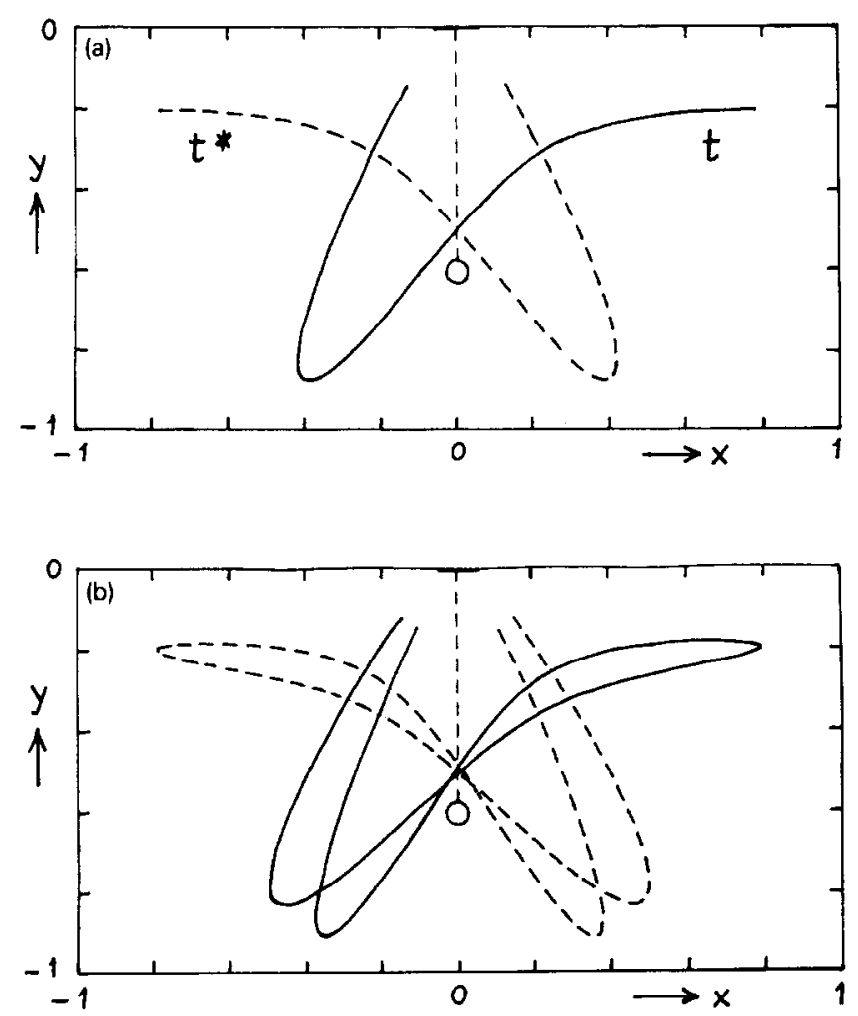

Fig. 7. (a) The oscillations $t$ and $t^{*}$, at $\mu=6, R=0.57$, just before period doubling. (b) The oscillations $\mathrm{t}$ and $\mathrm{t}^{*}$ at $\mu=6, R=0.66$, after the first period doubling bifurcation.

its twin oscillation $t^{*}$, just before (Fig. 7(a)) and just after (Fig. 7(b)) the first period doubling.

In Fig. 8 we follow the fate of a sea-star motion, lobsided to the left, and its twin motion (which is lobsided to the right) upon leaving the shaded region. These motions first recombine with each other to form one symmetrical orbit; this is shown in Figs. 8 (b) and 8(c). Eventually, the symmetrical orbit is squeezed away into the periphery of the phase portrait by the rotations $\mathrm{C}$ and $\mathrm{C}^{*}$, which become increasingly important as the energy grows. This course of events is typical for many motions.

Of course, one may also describe the order-chaos-order transition by more quantitative means, for instance by measuring the percentage of the available phase space which is covered by the chaotic orbits. This yiclds a percentage of (practically) zero in the limits $\mu \downarrow 1, \mu \rightarrow \infty, R \downarrow-1$ and $R \rightarrow \infty$ and a percentage of about $70 \%$ at the height of chaos in the intermediate region. We shall not pursue these percentages, interesting as they may be, but rather the dynamics behind them and in particular the changing dominance of the various main motions. This will be our task in the next sections. 


\section{The four limiting cases: order through decoupling}

\subsection{The limit $\mu \downarrow I$}

The limit $\mu \downarrow 1$ can be approached, though never really reached, by letting $k^{\prime} / m g=(\mu-1)$ become very small. In practice this can be done by taking a very weak spring ( $k$ small) and a large mass $m$. The equations of motion $(5 \mathrm{a}, \mathrm{b})$ can be written as:

$$
\begin{aligned}
& \ddot{x}=-\frac{k}{m} x+(\mu-1) g \frac{x}{\sqrt{x^{2}+y^{2}}}, \\
& \ddot{y}=-g-\frac{k}{m} y+(\mu-1) g \frac{y}{\sqrt{x^{2}+y^{2}}} .
\end{aligned}
$$

For small $(\mu-1)$ these are the equations for two weakly coupled oscillators. In the limit $\mu=1$ the coupling vanishes and the spring pendulum simply becomes an isotropic harmonic oscillator, with smooth elliptic orbits. In this limit, then, the motion is manifestly not chaotic.

One word about the isotropy for $\mu=1$ is perhaps in order. Naturally, when the $x$ and $y$ directions are decoupled the $x$ direction should get the pendulum frequency $\omega_{\mathrm{p}}$, and the $y$ direction the spring frequency $\omega_{\mathrm{s}}$, so at first sight it may seem strange to have $k / m$ in both directions. The reason is nevertheless very simple: the spring and pendulum frequencies, which are related by $\omega_{s}^{2}=k / m=\mu\left(\omega_{\mathrm{p}}^{2}\right.$, become identical in the limit $\mu=1$.

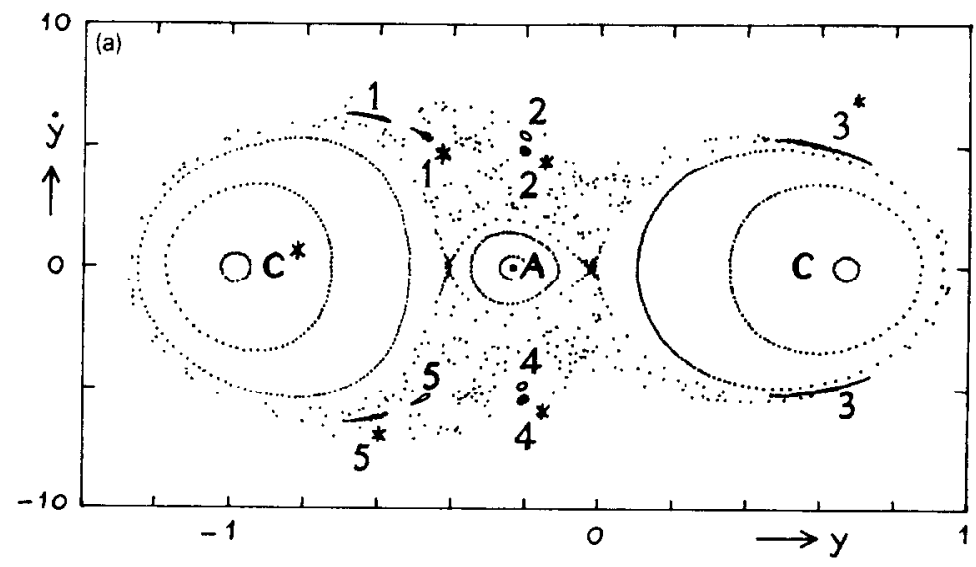

Fig. 8. (a) Poincaré section at $\mu=6, R=3.79$, showing the positions in phase space of two lobsided sea-star motions, which trace out the points 15 and $1^{*} 5^{*}$, respectively. (b) The shape of the same motions in the ( $x, y)$ plane. (c) The symmetrical sea-star motion at $\mu=6, R=5.68$, which has resulted from the recombination of the two lobsided stars. 

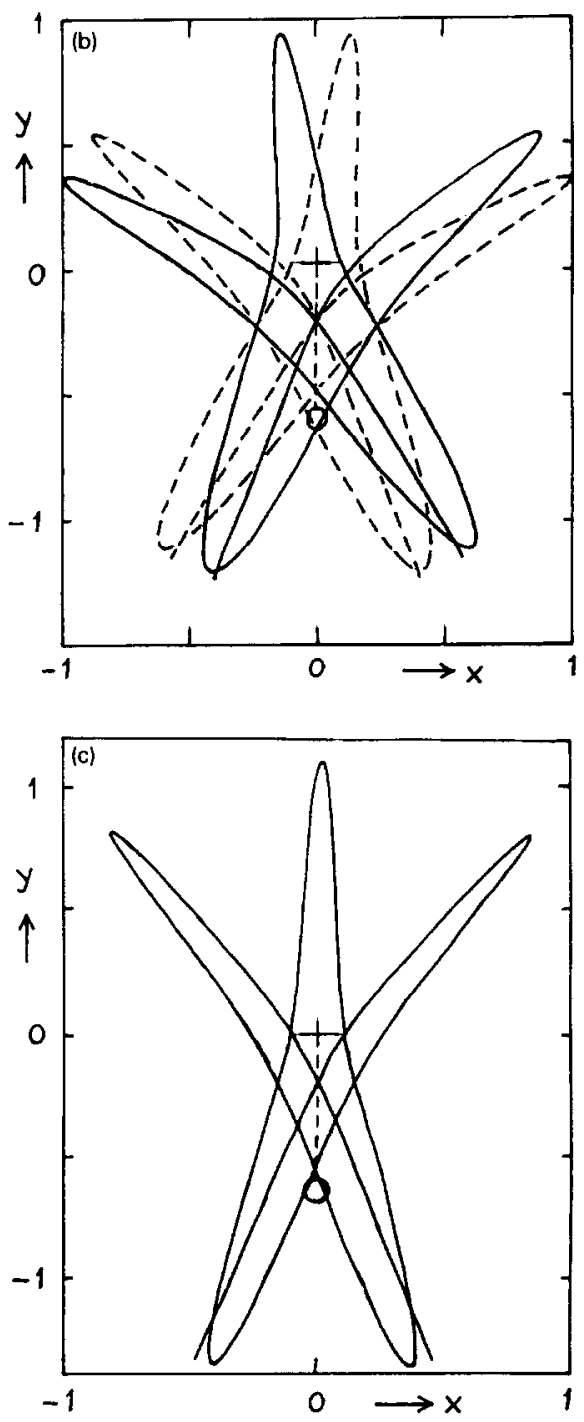

Fig 8. Continued.

\subsection{The limit $\mu>\infty$}

The limit $\mu \rightarrow \infty$, or equivalently $\left(k \ell_{0} / m g\right) \rightarrow \infty$, is most conveniently taken by letting the spring constant $k$ become infinite, which means that the spring is transformed into a rigid rod, with $r=\left(\ell_{0}+m g / k\right) \downarrow \ell_{0}$. This leaves us with a simple pendulum of length $\ell_{0}$. This can also be shown directly from the Lagrangian (4), or better still from the Lagrangian in polar coordinates (see also the Appendix):

$$
L=\frac{1}{2} m\left(\dot{r}^{2}+r^{2} \dot{\varphi}^{2}\right)+m g r \cos \varphi-\frac{1}{2} k\left(r-\ell_{0}\right)^{2} .
$$


In the limit $k \rightarrow \propto$ the motion in the radial direction is suppressed, so $r=0$ and $r=f_{0}$. The Lagrangian then reduces to

$$
L=\frac{1}{2} m \ell_{0}^{2} \dot{\varphi}^{2}+m g \ell_{0} \cos \varphi .
$$

This is indeed the Lagrangian of a mathematical pendulum, which is an integrable system. So also in this limit the motions of the spring pendulum are not chaotic. The main motions which play a role in this limit are of course $C_{\text {ose }}$ (below $R=1$ ) and $\mathrm{C} / \mathrm{C}^{*}$ (above $R=1$ ).

\subsection{The limit $R \downarrow-I$}

In contrast with the other limiting cases, the limit for small energies is quite connmonly encountered in practice. For $R \downarrow-1$, i.e. for energies just above $E_{\min }=-m g\left(t_{0}+m g / 2 k\right)$, the first motion to emerge is nothing but a small excursion from the downward equilibrium. For $\mu<4$ this happens to be an $\mathrm{A}$ oscillation (hill-shaped) and for $\mu>4$ a B oscillation (valley-shaped), and $\mu=4$ is a special case altogether. We shall briefly discuss these things.

Since we will be dealing with small excursions from the equilibrium position it is natural to use a coordinate system with this position in the origin; that is, instead of $(x, y)$ we now take coordinates $(x, z)$ with $z=y+\left(f_{0}+m g / k\right)$. The level of zero gravitational energy is also being put at $z=0$. In terms of the $x$ and $z$ coordinates the Lagrangian then reads

$$
L=\frac{1}{2} m\left(\dot{x}^{2}+\dot{z}^{2}\right)-m g z-\frac{1}{2} k\left(\sqrt{x^{2}+\left(z-\left(t_{0}+m g / k\right)\right)^{2}}-t_{0}\right)^{2}
$$

and expanding this in terms of $x$ and $z$ (since we are dealing with small excursions) this yields up to third order [7]:

$$
L=\frac{1}{2} m\left(\dot{x}^{2}-\omega_{\mathrm{p}}^{2} x^{2}\right)+\frac{1}{2} m\left(\dot{z}^{2}-\omega_{\mathrm{s}}^{2} z^{2}\right)+\frac{1}{2} m \dot{i} z x^{2}
$$

with

$$
i=\frac{k t_{0}}{m\left(/_{0}+m g / k\right)^{2}}
$$

The corresponding equations of motion are:

$$
\ddot{x}+\omega_{\mathrm{p}}^{2} x=\lambda x z \text { and } \ddot{z}+\omega_{s}^{2} z=\frac{1}{2} \lambda x^{2} .
$$

The form of these equations shows that we have to do with two harmonic oscillators, with frequencies $\left(\omega_{\mathrm{p}}\right.$ in the $x$ direction and $\omega_{\mathrm{s}}$ in the $y$ direction, and with a nonlinear coupling between them. It is this coupling which brings about the non-integrability but, being of higher order, it is almost zero for small values of $x$ and $z$. This means that there will be no chaos in this limit.

The motions are organized around the oscillations $A$ and $B$, in the shape of a hill and a valley, respectively; the hill is preferred for $\mu<4$ and the valley for $\mu>4$. This is illustrated by the Poincaré sections in Fig. 9 , at $\mu=3,4$ and 5, with $R=-0.92$ fixed. 

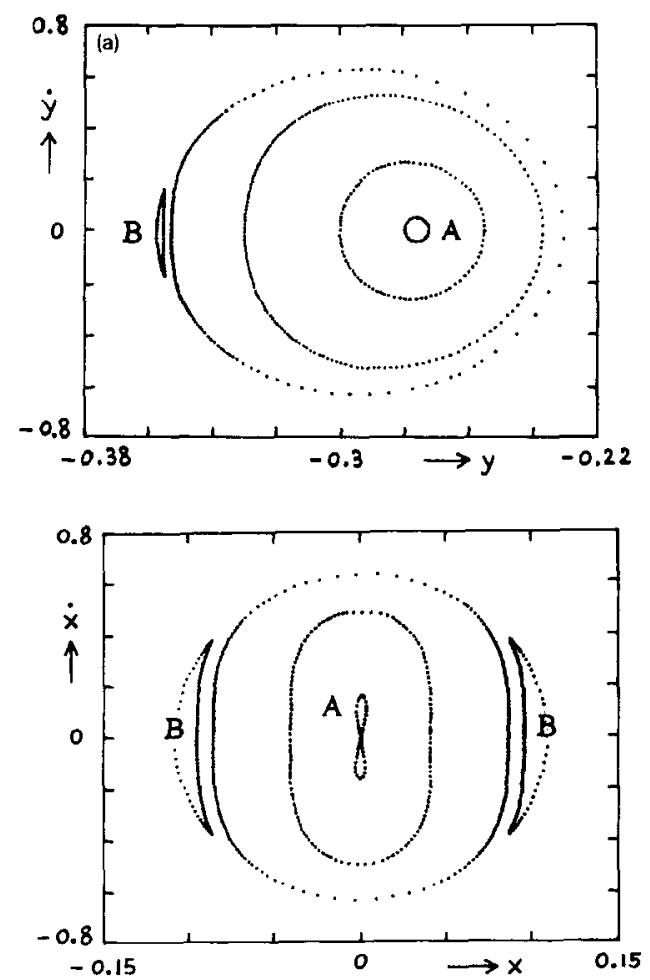

Fig. 9. Poincare sections through the phase space of the spring pendulum, at (a) $\mu=3$, (b) $\mu=4$ and (c) $\mu=5$, while $R=-0.92$ in all three cases. We show the $(y, y)$ plane, at the moments when $x$ goes through zero with $\dot{x} \geqslant 0$, as well as the $(x, \dot{x})$ plane at the moments when $y$ goes through a minimum (with $\hat{y}=0$ ).

In the $(y, \dot{y})$ sections the A oscillation corresponds to the right center point, and the $B$ oscillation to the one on the left. The transition from the one to other is evident. In practice one can easily see this changing preference by starting out from the vertical spring mode with a very small amplitude. In the $(y, \dot{y})$ sections this is difficult to follow, since the vertical spring mode docs not show up in these plots and for this reason we have also included sections through the $(x, \dot{x})$ plane; these have been obtained by plotting $(x, \dot{x})$ each time when $y$ goes through a minimum (where $\dot{y}=0$ ). The vertical spring mode corresponds with the origin of these sections; in each of the three cases this is a saddle point, reflecting the instability of the spring mode. For $\mu<4$ the vertical spring mode is excited into an A oscillation; at $\mu=4$ there is no preference for either A or B and the spring mode is excited into a (more or less pure) pendulum mode, which lies midway between $A$ and $B$; for $\mu>4$ the spring mode is excited into a $\mathbf{B}$ oscillation. The changing preference from $\mathbf{A}$ to $\mathbf{B}$ has been studied in detail elsewhere $[12,13]$ and can be explained loosely as follows: the spring pendulum prefers to oscillate in such a way that the spring goes up and down twice while the 

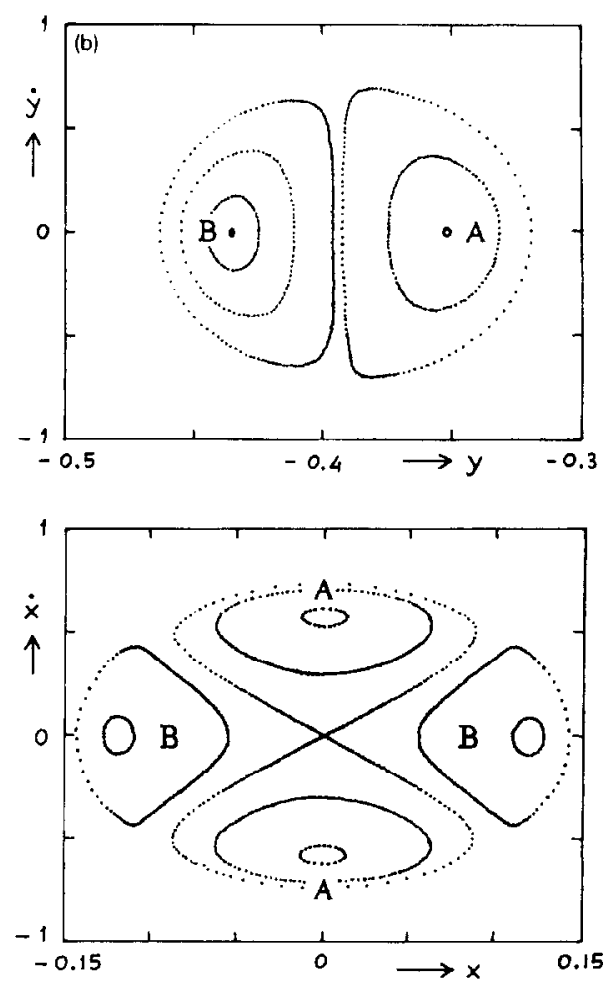

Fig 9. Continued.

pendulum goes back and forth once (a 2:1 Lissajous orbit). At $\mu-4$ the conditions are perfect for this, since the spring frequency $\left(\omega_{\mathrm{s}}\right.$ is exactly twice the pendulum frequency $\omega_{\mathrm{p}}$; the corresponding Lissajous figure looks like an infinity sign. For $\mu<4$ the spring is slow, but by adjusting its phase relative to the pendulum in such a way as to produce a hill oscillation (A) it is sped up and can still maintain a $2: 1$ orbit. For $\mu>4$ the spring is fast, but by adjusting its phase in such a way as to form a valley oscillation (B) it is slowed down and can again maintain a 2:1 orbit. The fact that a hill oscillation is faster than a normal pendulum mode, and a valley oscillation slower, is fairly easy to show [7]. At $\mu=4$, and as long as the amplitudes are sufficiently small, the A and B motions are well approximated by $x(t)=a \sin \omega t$ and $z(t)= \pm b \cos 2 \omega t+c$. Substituting these expressions into the equations of motion $(16 \mathrm{a}, \mathrm{b})$ and requiring that the lowest harmonics balance one obtains $b=a / 2 \sqrt{2}$, $c=3 a^{2} / 16 f_{\mathrm{c}}$ and

$$
(1)=()_{\mathrm{p}}\left(1 \pm \frac{3 \sqrt{2}}{16 t_{\mathrm{c}}} a\right)
$$



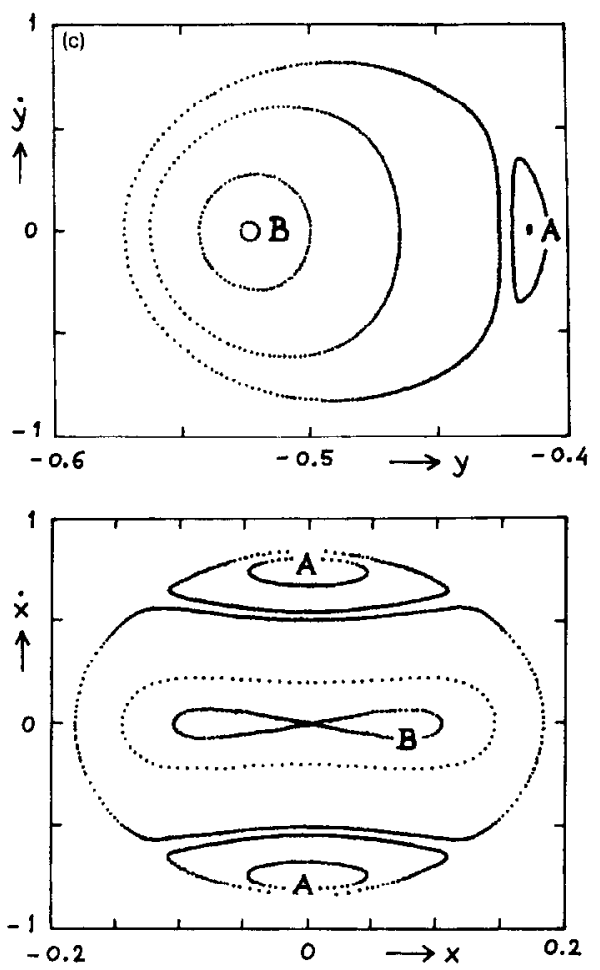

Fig 9. Continued.

where the ' + ' corresponds to the hill oscillation (A) and the ' - ' to the valley (B). We shall come back to the changing preference from A to B in Section 5.2.

Here we shall focus on another interesting aspect of the above Poincare sections, namely the fact that the saddle structure of the spring mode (the origin in the $(x, \dot{x})$ sections) becomes suddenly very large at $\mu=4$. This is the famous autoparametric resonance. To see how this comes about we take the equations of motion and start with an almost vertical oscillation of the form $z=a \cos \left(\omega_{\mathrm{s}} t\right)$ (the spring mode), which in practice will always be slightly perturbed of course in the horizontal direction. The motion in the $x$ direction is then governed by

$$
\ddot{x}+\left(\omega_{\mathrm{p}}^{2}-\lambda a \cos \left(\omega_{\mathrm{s}} t\right)\right) x=0 .
$$

This is a Mathieu equation, for which it is known that the solution $x=0$ is unstable for an interval of frequencies around $\omega_{\mathrm{s}}=2 \omega_{\mathrm{p}}$, which widens as $\lambda$ increases. (Mathematically speaking, the solution $x=0$ is unstable around $\omega_{\mathrm{s}}=(2 / n) \omega_{\mathrm{p}}$ for any value $n=1,2,3, \ldots[14]$ but since the spring pendulum is such that $\omega_{\mathrm{s}}>\omega_{\mathrm{p}}$, the only acceptable value is $n=1$.) So the spring mode will excite an oscillation in the $x$ direction if $\omega_{\mathrm{s}} \cong 2 \omega_{\mathrm{p}}$ or equivalently $\mu \cong 4$. For all other values of $\mu$ the spring mode will remain close to a spring mode, or, in other words, the saddle structure of the origin in the $(x, \dot{x})$ sections is small. 
If, vice versa, we start with an oscillation in the horizontal direction $x=a \cos \left(\omega_{\mathrm{p}} t\right)$ (the pendulum mode), with a slight vertical perturbation of course, the motion in the $z$ direction is governed by:

$$
\ddot{z}+\omega_{s}^{2} z=\frac{1}{2} \lambda a^{2} \cos ^{2} \omega_{\mathrm{p}} t=\frac{1}{4} \lambda a^{2}\left(1+\cos 2 \omega_{\mathrm{p}} t\right) .
$$

This describes a driven harmonic oscillator, with natural frequency $\omega_{\mathrm{s}}$ and a driving frequency $2 \omega_{\mathrm{p}}$. Resonant excitation occurs when these frequencies are (approximately) equal. So the pendulum mode will excite an oscillation in the $z$ direction if $\omega_{\mathrm{s}} \cong 2 \omega_{\mathrm{p}}$, or $\mu \cong 4$. This is the same condition as above (only the mechanism is different) and therefore the spring pendulum will continuously go back and forth between the two modes at $\mu \cong 4$.

In fact, the autoparametric resonance is a very ingenious coupling mechanism, since it succeeds in connecting the spring and pendulum modes (and quite strongly too) even though the coupling term is vanishingly small in the limit for $R \downarrow-1$. We shall come back to it in Section 5.2.

\subsection{The limit $R \rightarrow \infty$}

In the limit $R \rightarrow \propto$, or equivalently $E \rightarrow \infty$, the behaviour of the spring pendulum is organized around two equivalent circular rotations $\left(C\right.$ and $\left.C^{*}\right)$, one clockwise and the other counter-clockwise. For finite values of $R$ these rotations are of course only nearly circular (unless $\mu=\propto$ ) due to the gravitational force which tends to pull the spring pendulum downwards. The satellite motions around $\mathrm{C}$ and $\mathrm{C}^{*}$ take the form of a "rosette" as in Fig. 10.

In order to understand this rosette-shape it is instructive to consider the expression for the energy (in polar coordinates, as given in the Appendix):

$$
E=\frac{1}{2} m\left(\dot{r}^{2}+r^{2} \dot{\varphi}^{2}\right)-m g r \cos \varphi+\frac{1}{2} k\left(r-\ell_{0}\right)^{2} .
$$

In the limit $E \rightarrow \propto$ we may neglect the gravitational term $(-m g r \cos \varphi)$ and we get

$$
\begin{aligned}
E & \approx \frac{1}{2} m\left(\dot{r}^{2}+r^{2} \dot{\varphi}^{2}\right)+\frac{1}{2} k\left(r-t_{0}\right)^{2} \\
& =\frac{1}{2} m\left(r^{2}+r^{2} \dot{\varphi}^{2}\right)+\frac{1}{2} k r^{2}-k r f_{0}+\frac{1}{2} k t_{0}^{2} .
\end{aligned}
$$

The kinetic term and $\frac{1}{2} k r^{2}$ together constitute an isotropic two-dimensional oscillator and the term $-k r f_{0}$ can be viewed as a perturbation to this. The last term $\left(\frac{1}{2} k f_{0}^{2}\right)$ is a harmless constant. Now, it is a well-known fact that the (unperturbed) harmonic oscillator gives rise to closed, elliptic orbits with the geometrical centre of the ellipse coinciding with the centre of force. The perturbation $-k r \%_{0}$ causes the ellipses not to close upon themselves anymore; the result is a rosette, as in Fig. 10.

The energy in this limit is too large for oscillatory motions; these have ceased to exist $\left(\mathrm{C}_{\text {ose }}\right)$ or occupy a negligible part of the phase portrait $(\mathrm{A}$ and $\mathrm{B}$ ) and do not play any practical role anymore. In the process also the parametric resonance, i.e the 


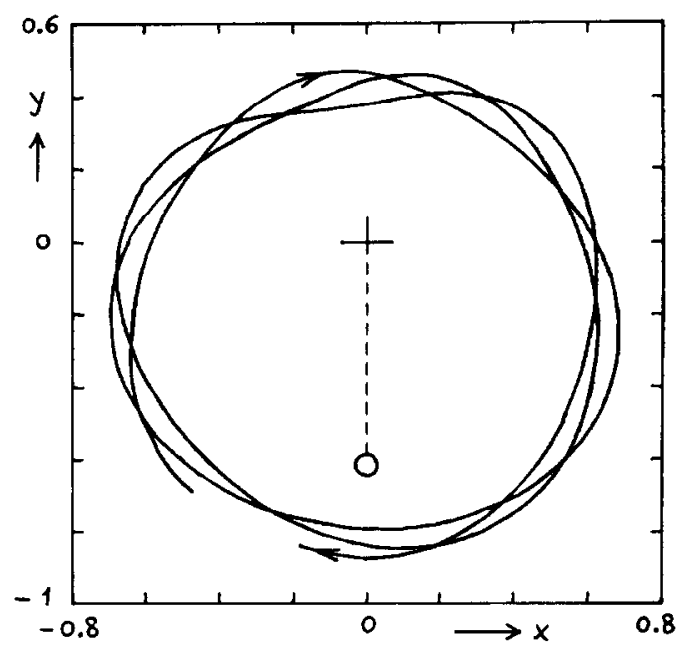

Fig. 10. The motion of the spring pendulum for large energy. The orbit depicted is a satellite motion of the C rotation.

special coupling between the horizontal and vertical modes, is nullified. So the value $\mu=4$ loses its significance at large values of $R$.

There is one other point which we would like to mention. Intuitively one might expect, in this limit $E \rightarrow \infty$, that the kinetic energy due to the rotational motion would give the dominant contribution to the total energy, but in fact the potential energy of the spring (which is stretched as a consequence of the centrifugal force) is of the same order of magnitude. This follows simply from the fact that for a (nearly) circular orbit the kinetic energy is given by

$$
\frac{1}{2} m r^{2} \dot{\varphi}^{2}=\frac{1}{2} k r\left(r-\ell_{0}\right)
$$

and the potential energy of the spring is $\frac{1}{2} k\left(r-\ell_{0}\right)^{2}$. Evidently, both expressions depend quadratically on $r$ and for $r \gg \ell_{0}$ they even become identical.

\section{The intermediate region: chaos}

In the previous section we have seen that at the boundaries of the $(\mu, R)$ plane the spring pendulum is decoupled into two autonomous one-degree-of-freedom systems. Such systems are always integrable and can only yield regular behaviour; hence the absence of chaos. Conversely, when the two degrees of freedom are not decoupled the system is non-integrable and will have an appreciable amount of chaos. This is what happens in the middle region of the $(\mu, R)$ plane, and in this section we try to answer the question where exactly the chaos reaches its height. We do this by addressing the equivalent question: when are the main motions at their weakest, i.e. when is the size 
of their satellite regions in phase space minimal? It turns out that this happens when the system hesitates, so to say, between different types of motion; and there are two instances when this occurs. One is associated with the transition from oscillations to rotations (or more precisely, from $\mathrm{C}_{\text {ose }}$ to $\mathrm{C} / \mathrm{C}^{*}$ ) and the other with the transition from hill-shaped (A) to valley-shaped (B) oscillations. We shall start with the transition from oscillations to rotations.

\subsection{Oscillations and rotations}

From the Poincare sections of Fig. 4 it is clear that the motion of the spring pendulum is restricted to one or two elliptical regions in the $(y, \dot{y})$ plane. The region for $y<0$ corresponds to oscillatory motions and counter-clockwise rotations, and the region for $y>0$ (which exists only above a certain threshold energy) corresponds to clockwise rotations. At high energy values the two regions coalesce. All this can be understood from cxpression (6) for the energy $E$.

Within the Poincare section we have $x=0$ and $\dot{x} \geqslant 0$, and at the houndary of the allowed region the energy due to the velocity $\dot{x}$ will be minimal, i.e. $\dot{x}=0$. Substituting $x=0$ and $\dot{x}=0$ in Eq. (6) we get:

$$
E=\frac{1}{2} m \dot{y}^{2}+m g y+\frac{1}{2} k\left(\sqrt{y^{2}}-f_{0}\right)^{2} .
$$

We now distinguish two cases: $y<0$ and $y>0$. In the case $y<0$ the above expression becomes:

$$
\begin{aligned}
E & =\frac{1}{2} m \dot{y}^{2}+m g y+\frac{1}{2} k\left(-y-\ell_{0}\right)^{2} \\
& =\frac{1}{2} m \dot{y}^{2}+\frac{1}{2} k\left(y+t_{0}+\frac{m g}{k}\right)^{2}-m g\left(t_{0}+\frac{1}{2} \frac{m g}{k}\right)
\end{aligned}
$$

which can also be written as follows:

$$
\frac{k\left(y+t_{0}+m g / k\right)^{2}}{2\left(E E_{\min }\right)}+\frac{m \dot{y}^{2}}{2\left(E-E_{\min }\right)}=1 .
$$

This form reflects the fact that the shape of the boundary is elliptical, centered around the point $(y, j)-\left(-\left(f_{0}+m g / k\right), 0\right)$ corresponding to the equilibrium position. The elliptic region reduces to this point when the denominator becomes zero, i.c. when $E=E_{\min }$. In the $(\mu, R)$ plane this is just the line $R=-1$.

For $y>0$, corresponding to rotations, expression (23) becomes:

$$
\begin{aligned}
E & =\frac{1}{2} m \dot{y}^{2}+m g y+\frac{1}{2} k\left(y-t_{0}\right)^{2} \\
& =\frac{1}{2} m \dot{y}^{2}+\frac{1}{2} k\left(y-t_{0}+\frac{m g}{k}\right)^{2}+m g\left(t_{0}-\frac{1}{2} \frac{m g}{k}\right)
\end{aligned}
$$

which can be written in the elliptical form:

$$
\frac{k\left(y-t_{\mathrm{o}}+m g / k\right)^{2}}{2\left(E-E_{\mathrm{up}}\right)}+\frac{m \dot{\nu}^{2}}{2\left(E-E_{\mathrm{up}}\right)}=1 .
$$


Here $E_{\mathrm{up}}$ is given by:

$$
E_{\text {up }}=m g\left(\ell_{0}-\frac{1}{2} \frac{m g}{k}\right) \text {. }
$$

This is the energy value corresponding to the spring pendulum in the upside down position (an unstable equilibrium) with $x=\dot{x}=\dot{y}=0$ and $y=\ell_{0}-m g / k$. It should be noted that this position only makes sense if $\ell_{0}>m g / k$, or $\mu>2$, because otherwise it would lie below the origin.

Eq. (27) represents the elliptical boundary for the clockwise rotational motions. For $\mu>2$ the boundary shrinks to zero if the denominator becomes zero at $E=E_{\mathrm{up}}=-\left(E_{\min }+(m g)^{2} / k\right)$. This yields a line in the $(\mu, R)$ plane below which no rotations are possible:

$$
R_{\mathrm{rot}}=1-\frac{2}{2(\mu-1)+1} \text { for } \mu>2
$$

The form of the minimal rotation, corresponding to $E_{\mathrm{up}}$, is sketched in Fig. 11(a) (dotted curve). It should be noted that this motion, on the borderline between oscillations and rotations, is structurally unstable. It is to be compared with the separatrix motion of a normal, nonextensible pendulum, which starts with zero velocity in the upright position, makes one revolution and returns (with zero velocity again) to the upright position. For such a pendulum the minimal energy is $-m g t_{0}$ and the upright position corresponds to an energy $m g t_{0}$, and consequently $R_{\text {rot }} \equiv-E_{\mathrm{up}} / E_{\min }=1$. In our system this situation is recovered in the limit $\mu \rightarrow \infty$.

This state of affairs is somewhat altered for $1<\mu<2$. In that case the minimal energy needed for a rotation is $E=\frac{1}{2} k t_{0}^{2}$. The origin then takes the place of the upright position. So now the minimal rotation is one with its top at the origin, and in fact looks more like a vertical oscillation. This motion turns out to be even more unstable than the minimal rotation for $\mu>2$. And admittedly it is a somewhat artificial motion, since at the moment of passage through the origin the spring pendulum is supposed to shrink to a point. Momentarily we then have $x=y=\dot{x}=\dot{y}=0$. In the $(\mu, R)$ plane this defines a curve

$$
R_{\mathrm{rot}}=\frac{(\mu-1)}{2+1 /(\mu 1)} \text { for } 1<\mu<2 .
$$

At $\mu=2$ (or $k \ell_{0}=m g$ ) the two curves (29) and (30) join smoothly, yielding a continuous and differentiable borderline for rotations; see Fig. 12.

Now, in the energy regime around $R_{\text {rot }}$ the main motions of the $\mathrm{C}$ type, i.e. the oscillation $\mathrm{C}_{\text {osc }}$ for $R<R_{\text {rot }}$ and the rotations $\mathrm{C} / \mathrm{C}^{*}$ for $R>R_{\text {rot }}$, are too weak to act as organizing centers of the dynamics. They are simply too close to the minimal rotations involving the unstable upright position. This is corroborated by the Poincarc scctions in Fig. 4(c) and (d), where it is seen that the island of satellite motions 

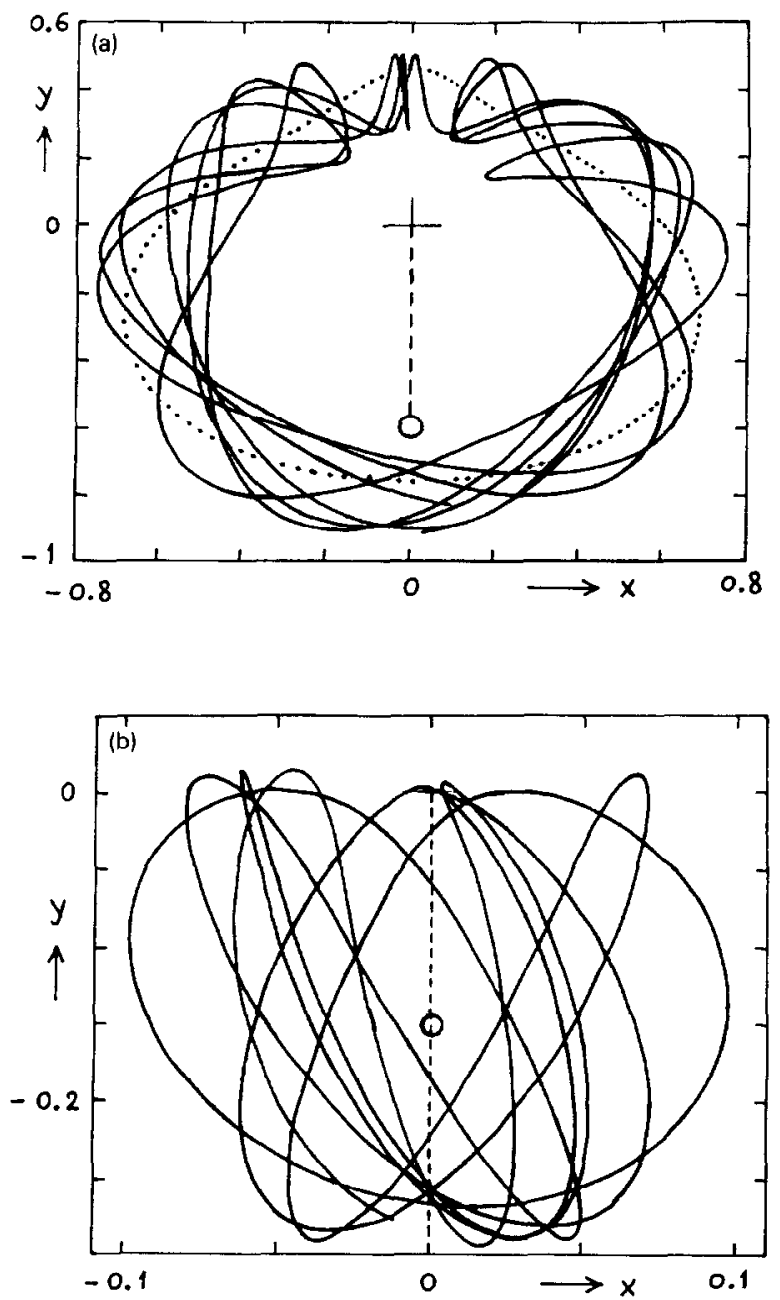

Fig. 11. (a) Near the minimal rotation for $\mu=6$, at $R_{\mathrm{r} m}=0.818$. The actual minimal rotation (dotted curve) is unstable. The solid curve is the trajectory of a nearby motion. an erratic sequence of rotations and oscillations. (b) Ncar the minimal rotation for $\mu=1.5$, at $R_{\text {rot }}=0.125$. The actual minimal rotation, or rather oscillation (along the vertical dashed line), is unstable. The solid curve is the trajectory of a chaotic nearby motion. This motion is actually closer to the vertical oscillation than it appears, since the horizontal scale is stretched to make the picture less crammed.

around point $C$ becomes very small. In fact, at the transition energy $R_{\mathrm{rot}}$ (where the oscillation $\mathrm{C}_{\text {osc }}$ and the rotations $\mathrm{C} / \mathrm{C}^{*}$ are transformed into each other) the island momentarily reduces to a single point. This is what makes the curve $R_{\text {rot }}$ a backbone of chaos in the $(\mu, R)$ plane.

In the same figures $(4(\mathrm{c})$ and $(d))$ we observe that the valley-shaped $B$ oscillation is being pushed to the edge of the phase portrait for growing $R$, and that its satellite 
region has become already very narrow at $R_{\text {rot }}$. Only the hill-shaped A oscillation is still relatively strong, but this is not enough to suppress the chaos anymore.

\subsection{The role of parametric resonance}

So, in the $R$ direction chaos is concentrated around the curve $R_{\text {rot }}$, where the spring pendulum hesitates between oscillations and rotations. In the $\mu$ direction the line $\mu=4$ plays a similar role; here the system hesitates between the $\mathrm{A}$ and B oscillations, as we have seen in Section 4.3. Another way of looking at the same thing is to recognize that the parametric resonance at $\mu=4$, which so ingeniously couples the two degrees of freedom of the system, is a natural source of chaos. After all, any chaos in the spring pendulum exists by virtue of the coupling of the two degrees of freedom.

These two characteristics of $\mu=4$ are beautifully reflected by the Poincare sections in Fig. 9. The changing preference from $\mathrm{A}$ (for $\mu<4$ ) to $\mathrm{B}$ (for $\mu>4$ ) is evident from the $(y, \dot{y})$ as well as from the $(x, \dot{x})$ sections, and the strong coupling between the two degrees of freedom at $\mu=4$ is reflected by the magnitude of the saddle structure in the corresponding $(x, \dot{x})$ section. In each of the three sections we have a saddle point at the origin, showing that the vertical spring mode is unstable, but while at $\mu=3$ and $\mu=5$ the influence of this saddle point is restricted to the immediate vicinity of the origin (for all practical purposes the spring mode just remains a spring mode) we see that at $\mu=4$ the saddlepoint connections throw a cross over the complete available phase space. Since saddlepoint connections are, by their complex web of intersections, responsible for chaos we conclude that the phase space of the spring pendulum at resonance is contaminated with chaos right to the edges in one single stroke.

This makes the line $\mu=4$ another backbone of chaos, or at least the lower part of this line. Above the line $R_{\text {rot }}$ the $\mathrm{A}$ and $\mathrm{B}$ oscillations still exist, but their importance swiftly diminishes as the dynamics becomes more and more dominated by the rotations $\mathrm{C} / \mathrm{C}^{*}$. The system's hesitation between $\mathrm{A}$ and $\mathrm{B}$ is then of no consequence anymore. That is why in Fig. 12 the line $\mu=4$ has not been extended to large values of $R$. In spite of its shortness, however, the $\mu=4$ backbone is by no means an unimportant one. This is because it lies in the midst of the most relevant region of the $(\mu, R)$ plane, from a practical point of view, with moderate $\mu$ values and relatively low $R$.

Summarizing, we see that chaos is concentrated in the $(\mu, R)$ plane around the curve $R_{\text {rot }}$ and the lower part of the line $\mu=4$, and therefore the core of any order-chaos-order transition lies there. The height of chaos is to be found at the intersection point of the two lines, i.e. at $(\mu, R)=\left(4, \frac{5}{7}\right)$. This is confirmed by our own numerical results (we have made a rather complete survey of the relevant region in the $(\mu, R)$ plane and compared the amount of chaos in the various Poincare sections) and also by those of Núñez-Yépez et al. [3]. These authors deal only with the case $\mu=4$ (or $f=0.25$ in their notation) and report that the relative amount of phase space 


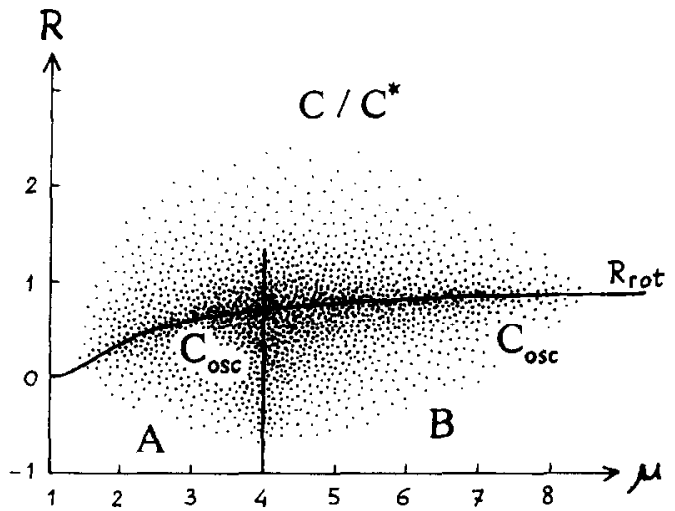

Fig. 12. The structure of the $(\mu, R)$ plane. Each main motion has its own region of dominance. The curve $R_{\text {rot }}$ is the line above which rotations are possible; below this line the spring pendulum can only perform oscillatory motions. The vertical line $\mu=4$ corresponds to parametric resonance.

occupied by irregular orbits attains its maximum at a certain energy value between $R=0.64$ and $R=0.85$ (or $E=0.42$ and $E=0.47$ in their notation). Our theoretical prediction $R=\frac{5}{7}=0.71$ (or $E=\frac{7}{16}=0.44$ ) lies perfectly within this interval.

\section{Discussion}

\subsection{Summary}

Our results are summarized in Fig. 12. The behaviour of the spring pendulum becomes regular in the limits $\mu \downarrow 1, \mu \rightarrow \infty, R \downarrow-1$ and $R \rightarrow \infty$. In the intermediate region one finds regular as well as chaotic behaviour, with chaos dominating in the neighbourhood of the dashed curves $R_{\text {rot }}$ (given by Eqs. (29) and (30)) and $\mu=4$. The regular motions are organized around four main motions, denoted by the letters $\mathrm{A}, \mathrm{B}, \mathrm{C}_{0 * \mathrm{c}}$ (all oscillations) and $\mathrm{C} / \mathrm{C}^{*}$ (rotation); these are depicted in $\Gamma$ ig. 5. As we have indicated in Fig. 12, each of the main motions has its own favourite region in the $(\mu, R)$ plane, although they are not strictly separated since all these motions (except $C_{0 * \mathrm{c}}$ and $\mathrm{C} / \mathrm{C}^{*}$ ) usually coexist. In between the favourite regions the spring pendulum "hesitates" between the various motion types, and that is where chaos takes over.

The line $\mu=4$ in Fig. 12 has not been drawn up to large values of $R$. $\Lambda$ bove the intersection with $R_{\text {rot }}$ the rotations $\mathrm{C} / \mathrm{C}^{*}$ soon become so dominant that the system's hesitation between the $\mathrm{A}$ and $\mathrm{B}$ oscillations is no longer of any consequence. The lower part of the line $\mu=4$ is all the more important, since this lies precisely in the region of the $(\mu, R)$ plane which is encountered most often in practice. 


\subsection{On the idealizations in our model}

For the sake of clarity and theoretical elegance we made a few idealizations in our model. We neglected dissipation, and we assumed that the spring was massless, but have we not strayed too far from reality by doing so?

Let us start with the dissipation. Thanks to the fact that we left it out of our model all motions were everlasting. Obviously, this quite unrealistic. Everybody knows that an experimental spring pendulum will sooner or later (but inevitably) come to a halt in its equilibrium rest position, due to air resistance, friction in the point of support, and other forms of energy dissipation. This makes a huge difference, but it is not so bad as it seems, and in fact the dissipation of the energy (while $\mu$ remains intact) may even be regarded as an advantage. It causes the system to follow a vertical, downward path through the $(\mu, R)$ parameter plane, eventually coming down to the point $(\mu,-1)$; thus, all one has to do is to give the spring pendulum a nearly circular rotation (or any other motion with sufficiently large energy), sit back and watch the order-chaos-order sequence take place for free. The only condition is that the dissipation should not be too strong, because otherwise the chaos is smothered and every trajectory is immediately attracted to one of the main types of motion. In that case one will witness the $C$ rotation transform into a $C_{\text {ose }}$ oscillation, which in its turn will give way to an $\mathrm{A}$ or $\mathrm{B}$ oscillation before the spring pendulum finally comes to rest in its downward equilibrium position.

The neglect of the spring mass is even less of a problem. True, for a real spring pendulum the frequency $\omega_{\mathrm{s}}$ will be somewhat smaller due to the spring mass $m_{\mathrm{s}}$, according to the relation [15]:

$$
\omega_{\mathrm{s}}=\sqrt{\frac{k}{m+\delta m_{s}}},
$$

where $\delta$ is a factor depending on the mass ratio $m_{\mathrm{s}} / m$, ranging from $0.333\left(=\frac{1}{3}\right)$ for $m_{\mathrm{s}} / m \downarrow 0$ to $0.405\left(=4 / \pi^{2}\right)$ in the limit $m_{\mathrm{s}} / m \rightarrow \infty$. This has its effect, of course, on the parameter $\mu$. For instance, while the theoretical spring pendulum shows parametric resonance $(\mu=4)$ if the mass $m$ is chosen such that $\ell_{\mathrm{e}}=\frac{4}{3} \ell_{0}$, a real spring pendulum will be resonant $(\mu=4)$ at a smaller mass $m$ and hence at $\ell_{\mathrm{e}}<\frac{4}{3} \ell_{0}$. But, evidently, this is a rather harmless difference. Another consequence of the spring mass is that the spring admits, besides the fundamental mode with frequency $\omega_{\mathrm{s}}$, also higher normal modes with higher frequencies [16-19]. Luckily, however, the amplitudes of these higher normal modes can be shown to be too small (for all values of $m_{\mathrm{s}} / m$ ) to be significant [17]. So, all things considered, the idealizations in our model are really not too bad.

By the way, the above two idealizations were not the only ones. An experimental spring pendulum will usually not be restricted to just one plane, and will really be a spherical spring pendulum. Furthermore, the spring will also twist a little bit around its vertical axis; when the spring shortens it is winded a little bit tighter, and when it 
lengthens it is unwinded again. That is, there will be some torsion. All these subtleties do not change the main point, however, and therefore (just as the dissipation and the spring mass above) they are better left out of the model.

\subsection{Similar sequences in other systems}

Now that we understand the spring pendulum, let us finally examine how representative this system is for order chaos-order sequences in general. To this end we compare it with a few other examples which have been documented in the literature.

To begin with, Deng and Hioe [6] studied two nonlinearly coupled nonlinear oscillators:

$$
\ddot{x}+x+4 \lambda x^{3}=-4 \lambda C x y^{2}, \quad \ddot{y}+y+4 \lambda y^{3}=-4 \lambda C x^{2} y,
$$

where $\lambda$ is a scaling parameter and $C$ represents the coupling strength. They found a chaos-order-chaos sequence when they varied $C$ from $-\infty$ to $x$, with chaos for $C \leqslant-0.21$ and $C \geqslant 5.2$, and regular behaviour in between. This is different from the case of the spring pendulum in two respects. In the first place the succession of the sequence is different, with order in the middle instead of at the endpoints, and secondly the transitions from chaos to order and vice versa seem to be much more abrupt.

Reichl and Büttner [1] investigated a system of two Toda oscillators (each representing an integrable two-particle system), connected with each other via a nonlinear coupling term. The two parameters of this system, the total energy $E$ and the coupling strength $C$, were varied independently and in both cases an order-chaos order sequence was observed, very much like that of the spring pendulum. Similar behaviour, but with a smaller stochastic region, was found when the Toda oscillators were replaced by harmonic oscillators.

Bolotin et al. [2] studied the following generalized Hénon-Heiles system:

$$
\begin{aligned}
& \ddot{x}+a x+4 c x^{3}=-2 b x y-4 c x y^{2} \\
& \ddot{y}+a y-b y^{2}+4 c y^{3}=-b x^{2}-4 c x^{2} y,
\end{aligned}
$$

which is formally equivalent with two nonlinear, nonidentical oscillators, connected by a nonlinear and nonsymmetrical coupling. If one varies the total energy of this system at a fixed value of $W \equiv b^{2} / a$ (in the range from 4 to 16), as the authors did, one finds an order-chaos-order sequence. It is not completely clear from their paper how abrupt the transitions in this sequence are, but in all other respects it resembles the case of the spring pendulum.

Actually, this is no wonder, since the spring pendulum falls within the same class of nonlinearly coupled oscillators. It consists of two dissimilar oscillators, the one linear (the spring) and the other nonlinear (the pendulum), coupled to each other in a nonlinear, non-symmetrical way. From a mathematical point of view it is certainly 
not the most economic model; the coupling does not have to be nonsymmetric and the oscillators need not be different, probably not even nonlinear (see Ref. [2]). On the other hand, from a physicist's point of view, the spring pendulum is perhaps the most elegant and straightforward example of all.

So, the spring pendulum may be taken to be representative for a whole class of coupled oscillator systems. But probably it is rather more general than that. After all, any system exhibiting an order-chaos-order sequence must be nonintegrable (since otherwise chaos is not possible in the first place) and for certain choices of the parameters the system should reduce to an integrable one (or otherwise there would be no absence of chaos). The way this is achieved in the spring pendulum, by the decoupling of the various degrees of freedom and thus separating the system into (integrable) one-degree-of-freedom systems, is so natural that it is hard to imagine any other way. As far as we can see there are only two aspects of the spring pendulum's sequence which are not really representative. These are the smoothness of its transitions from order to chaos and vice versa, and the fact that it may convey the impression that the sequence always takes the form "order-chaos-order", while in fact we know (see Ref. [6]) that "chaos-order-chaos" is equally possible, and even exotic sequences such as "chaos-order-chaos-order-chaos" should not be ruled out.

\section{Acknowledgement}

The authors gladly seize the opportunity to congratulate Professor H.W. Capel on the occasion of his 60th hirthday.

\section{Appendix. The spring pendulum in polar coordinates}

In polar coordinates (the distance $r$ from the point of support, and the angle $\varphi$ from the downward vertical) the Lagrangian of the spring pendulum reads:

$$
L=\frac{1}{2} m\left(\dot{r}^{2}+r^{2} \dot{\varphi}^{2}\right) \perp m g r \cos \varphi \quad \frac{1}{2} k\left(r \quad \digamma_{0}\right)^{2},
$$

and the associated equations of motion take the form:

$$
\begin{aligned}
& \ddot{r}=r \dot{\varphi}^{2}+g \cos \varphi-\frac{k}{m}\left(r-\ell_{0}\right) \\
& \ddot{\varphi}=-2 \frac{\dot{r} \dot{\varphi}}{r}-\frac{g}{r} \sin \varphi .
\end{aligned}
$$

The total energy is:

$$
E=\frac{1}{2} m\left(\dot{r}^{2}+r^{2} \dot{\varphi}^{2}\right) \quad m g r \cos \varphi+\frac{1}{2} k\left(r \quad \ell_{0}\right)^{2} .
$$




\section{References}

[1] J. Reichl and H. Büttner. Stochastic and regular motion in a four-particle system. Phys. Rev. A 33 (1986) 21842185 .

[2] Yu. L. Bolotin, V. Yu. Gonchar, V.N. Tarasov and N.A. Chekanov, The transition "regularity-chaos-regularity" and statistical properties of energy spectra. Phys. Let1. A 135 (1989) $29 \cdots 31$.

[3] H.N. Núnez-Yépez, A.L. Salas-Brito, C.A. Vargas and L. Vicente. Onset of chaos in an extensible pendulum. Phys. Lett. A 145 (1990) 101-105.

[4] R. Carretero-González, H.N. Núñez-Yépez and A.L. Salas-Brito, Regular and chaotic behaviour in an extensible pendulum, Eur. J. Phys. 15 (1994) 139148.

[5] R. Cuerno, A.F. Rañada and J.J. Ruiz-Lorenzo, Deterministic chaos in the elastic pendulum: a simple laboratory for nonlinear dynamics, Am. J. Phys. 60 (1992) 7379

[6] Z. Deng and F.T. Hioe. Chaos-order chaos transitions in a two-dimensional Hamiltonian system. Phys. Rev. Lett. 55 (1985) 1539-1542.

[7] M.G. Olsson, Why does a mass on a spring sometimes misbehave?, Am. J. Phys. 44 (1976) 12111212.

[8] N. Minorsky. Nonlinear Oscillations (Van Nostrand, Princeton. NJ, 1962). The autoparametric excitation of the spring pendulum is discussed in Chap. 20.5, pp. 506-509.

[9] T.E. Cayton. The laboratory spring-mass oscillator: an example of parametric instability. Am. I. Phys. 45 (1977) 723732.

[10] L. Falk, Recurrence effects in the parametric spring pendulum, Am. J. Phys. 46 (1978) 1120-1123: see also L. Falk, Student experiments on parametric resonance. Am. J. Phys. 47 (1979) 325328.

[11] M.G. Rusbridge, Motion of the sprung pendulum, Am. J. Phys. 48 (1980) 146151.

[12] E. Breitenberger and R.D. Mueller. The elastic pendulum: a nonlinear paradigm, J. Math. Phys. 22 (1981) $1196 \quad 1210$.

[13] H.M. Lai. On the recurrence phenomenon of a resonant spring pendulum, Am. J. Phys. 52 (1984) $219-223$.

[14] N.W. McLachlan, Theory and Applications of Mathieu Iunctions (Dover, New York, 1964).

[15] E.O. Galloni and M. Kohen, Influence of the mass of the spring on its static and dynamic cffects, Am. J. Phys. 47 (1979) 10761078.

[16] R. Weinstock, Oscillations of a particle attached to a heavy spring: an application of the Stieltjes integral, Am. J. Phys. 47 (1979) 508-514

[17] J.T. Cushing, The spring-mass system revisited, Am. J. Phys. 52 (1984) 925933.

[18] J.T. Cushing. The method of characteristics applied to the massive spring problem. Am. J. Phys. 52 (1984) 933937.

[19] J.M. Nunes da Silva, Renormalized vibrations of a loaded spring, Am. J. Phys. 62 (1994) 423426. 